\title{
Clusters of Responders and Predictive Factors for Response to Supplementation with Boswellia, Turmeric, and Red Algae Extracts in Painful Knee Osteoarthritis: A Prospective Observational Study Using an Arsenal of Patient-Centered Measures
}

This article was published in the following Dove Press journal: Open Access Rheumatology: Research and Reviews

\author{
Samira Ait Abdellah' \\ Caroline Gal' \\ Anne Leblanc ${ }^{2}$ \\ Anne-Priscille Trouvin (iD ${ }^{3}$ \\ Serge Perrot ${ }^{3}$ \\ 'Clinical Research Department, PiLeje \\ Laboratoire, Paris, France; ${ }^{2}$ Applied \\ Research Department, PiLeje \\ Laboratoire, Paris, France; ${ }^{3}$ Pôle Ostéo- \\ Articulaire, Hôpital Cochin, Paris, France
}

Correspondence: Serge Perrot

Pôle Ostéo-Articulaire, Hôpital Cochin,

27 Rue Du Faubourg Saint-Jacques, Paris

75014 , France

Tel +33 I58 4II 507

Email serge.perrot@aphp.fr
Purpose: This observational study evaluated a combination of boswellia, turmeric, and red algae extracts in patients with knee osteoarthritis (KOA). Given the growing interest in patientcentered care in osteoarthritis, effects were assessed by an arsenal of patient-reported outcome measures (PROMs): Patient Acceptable Symptom Scale (PASS), Minimal Clinically Important Improvement (MCII), Patient Global Impression of Change (PGIC), and Lequesne algofunctional index (LAFI). Patients also completed a list of 17 items on pain quality.

Patients and Methods: Patients with painful unilateral or bilateral KOA had to take 1-4 capsules per day of a dietary supplement containing boswellia, turmeric, and red algae extracts for 90 days. Patients completed PROMs on Days 0 (baseline), 10, 20, 30, 60, and/or 90.

Results: A total of 118 patients [female: 69.5\%; age: 62.9 (9.5) years, mean (SD)] were included in the study and took at least one capsule. Mean (SD) follow-up duration was 100.7 (54.9) days. Pain relief was maximal on Day 90: 64.5\% of patients were responders (positive PASS); $68.8 \%$ and $58.4 \%$ had MCII and PGIC scores indicating positive effect (score $\geq 3$ ) or global improvement (score $\geq 5$ ); $73.3 \%$ (versus $47.5 \%$ at baseline) were mildly/moderately disabled (LAFI score $<8$ ); $55.2 \%$ had meaningful decrease $(-30 \%)$ in pain intensity (VAS), $35.1 \%$ (versus $59.2 \%$ at baseline) took analgesics as supplementary treatment. Median time to the first PASS change was 34 days. Pain intensity (VAS), as well as two pain characteristics (ie, "Stabbing pain" and "Widespread pain"), were independent factors associated with non-response on Day 30. Four clusters of responders were isolated according to pain characteristics, with one cluster exhibiting a higher responder rate.

Conclusion: The results of this preliminary study suggest that the combination of boswellia, turmeric, and red algae extracts tested could improve KOA patients. Beyond these results, this study showed the importance of PROMs and specific pain qualitative descriptors for the accurate evaluation of dietary supplement approaches in painful conditions.

Keywords: Curcuma, dietary supplement, joint disease, Porphyra, self-reported questionnaire

\section{Introduction}

Osteoarthritis (OA) is a common degenerative age-related disease involving movable joint, especially knees. ${ }^{1-3} \mathrm{OA}$ is a painful disabling chronic condition that limits activities of daily living and impacts quality of life. ${ }^{1,2}$ It is the source of nonnegligible socioeconomic costs. ${ }^{4}$ 
As the restoration of the imbalances between catabolic and anabolic processes that underlie cartilage pathogenesis is nearly impossible, knee osteoarthritis (KOA) remains incurable. The objective of KOA treatments is thus to relieve pain and maintain joint function. According to the most recent guidelines, core treatments for KOA include arthritis education and structured land-based exercise programs with or without dietary weight management. ${ }^{5}$ Considering pharmacological treatments, topical are preferred to oral non-steroidal anti-inflammatory drugs (NSAIDs) due to the possible occurrence of severe adverse events, especially in patients at gastrointestinal, renal, or cardiovascular risk. Moreover, given its limited efficacy and hepatic concerns, acetaminophen is not recommended. However, in clinical practice, oral NSAIDs and acetaminophen are frequently used. ${ }^{6}$ In a study interviewing patients with self-reported peripheral joint $\mathrm{OA}$ from France, Germany, Italy, Spain, and the United Kingdom, $50 \%$ of patients claimed to have a medical prescription, and in a French study interviewing patients asking for over the counter (OTC) analgesics in pharmacy, $50 \%$ of them claimed to have rheumatologic or musculoskeletal pain. ${ }^{7,8}$ That is why the development of alternative treatments to manage KOA without adverse events is crucial.

Traditional medicines using natural-derived compounds may offer this safe alternative. ${ }^{3}$ Numerous studies, including several randomized clinical trials versus placebo or NSAIDs, have shown that boswellia (Boswellia serrata) and turmeric (Curcuma longa) extracts (alone or in combination) may relieve patients. ${ }^{9-20}$ Their efficacy was supported by the presence of active ingredients with anti-inflammatory and/ or anti-nociceptive properties such as boswellic acids or curcumin. ${ }^{3,21-25}$ In addition, some in vitro and in vivo studies showed the anti-inflammatory and anti-nociceptive effects of sulphated-polysaccharides extracted from marine algae. ${ }^{26-29}$ Recently, PiLeJe Laboratory found that extracts of red algae (Porphyra umbilicalis) that contain more than $30 \%$ of sulphated-polysaccharides could relieve mice with kaolininduced arthritis (internal data not shown).

The objective of this preliminary study was to evaluate the effects of a dietary supplement combining extracts of boswellia, turmeric, and red algae in KOA patients. As the objective of patient-centered care is not to treat the patient "with what is satisfactory for most people but with what is personally meaningful for him or her", ${ }^{30}$ the effects of the dietary supplement were assessed by an arsenal of patientreported outcome measures (PROMs), including a list of pain descriptors.

\section{Patients and Methods Study Design and Ethics Statement}

This was an open-label, observational, longitudinal study performed in France. The study was conducted by general practitioners (GPs) who knew the dietary supplement, and usually recommend its use in the treatment of OA flareups, in addition to a balanced diet.

The study was approved by the Advisory Committee on Information Processing in Material Research in the Field of Health (Comité d'Ethique agreement no.: 16-634). It was performed in accordance with the ethical standards laid down in the Declaration of Helsinki and the Strengthening the Reporting of Observational studies in Epidemiology (STROBE) guidelines. The present study was registered on the ClinicalTrials.gov site in November 2016 under the following identifier number: NCT02977936.

\section{Participants and Enrolment}

Male and female adult ( $>18$ years) patients with unilateral or bilateral KOA (with flare-ups) could be included in the study if they reported pain $\geq 4$ at rest or $\geq 5$ on movement (walking) on a 10 -cm visual analogue scale (VAS) and were unsatisfied by their symptomatic state (negative PASS at the time of recruitment). The diagnosis of KOA was to be confirmed by a clinical examination and an Xray in the last 2 years (Kellgren-Lawrence radiographic severity grade of 2 or 3 ).

Conversely, the following patients could not be included in the study: (1) patients with non-OA rheumatological condition, (2) patients with chronic pain liable to interfere with KOA pain, (3) patients waiting for knee surgery within the three forthcoming months, (4) patients who started a chondroitin sulphate-based treatment (or any osteoarthritis treatment other than anti-inflammatory and analgesic drugs) less than a month ago, (5) patients with reduced mobility and bedridden, (6) patients with body mass index (BMI) $>35 \mathrm{~kg} / \mathrm{m} 2$, (7) patients with cognitive disorders preventing study participation, (8) patients with known hypersensitivity to at least one of the components of the dietary supplement, and (9) pregnant and breastfeeding women.

All compliant patients received written information about the study and gave their verbal consent before being included in the study in compliance with the French regulation for observational studies (approval by the Advisory Committee on Information Processing in Material Research in the Field of Health). 


\section{Supplement}

The dietary supplement was a fixed combination of boswellia, turmeric, and red algae extracts (Cartimotil Fort ${ }^{\mathbb{B}}$, PiLeJe Laboratoire, Paris, France). The dietary supplement was marketed in France in 2016. Each capsule contains $75 \mathrm{mg}$ of boswellia extract (Boswellia serrata L., bark), $175 \mathrm{mg}$ of turmeric extract (Curcuma longa L., roots), and $150 \mathrm{mg}$ of red algae extract (Porphyra umbilicalis). The dietary supplement was recommended to be taken at the dose of one to four capsules per day for 90 days (one per day in non-crisis situations and up to four in case of pain flare-up).

\section{Procedure}

At inclusion visit, after having checked the patient's eligibility, the GP completed for each patient an inclusion form including sociodemographic, anthropometric, and KOA characteristics. At the end of the visit, as they would have done in routine clinical practice, the GP recommended the dietary supplement.

Patients who took the dietary supplement had to complete several questionnaires, including some PROMs, and provide information on compliance and the use of concomitant antalgics or anti-inflammatory drugs to evaluate dietary supplement effectiveness and tolerance throughout the 90 days of dietary supplementation. All data were collected during an end-of-study visit performed approximately three months after the start of the study. Data were then captured on an electronic case report form (e-CRF).

\section{Main Evaluation Scales and Endpoints}

On Days 0, 10, 20, 30, 60, and/or 90, patients completed three single-item tools: the Patient Acceptable Symptom Scale (PASS), the Minimal Clinically Important Improvement (MCII), and the Patient Global Impression of Change (PGIC). They also completed the Lequesne algofunctional index (LAFI), a multiple-item tool, and a list of items assessing pain quality.

The PASS assesses the ability of a treatment to bring patients to an acceptable and stable symptomatic state. It is a 2-point scale, with the options acceptable (positive) or nonacceptable (negative). ${ }^{31}$ The exact wording of the question is as follows:

Taking into account all the activities you have in your daily life, your level of pain and also your functional disability, if you were to remain in your condition for the next few months, would you consider your current state to be acceptable? Yes/No.
By convention, only patients with negative PASS at inclusion could be included in the study, and patients were considered responders if they had a positive PASS during the dietary supplementation period. The MCII evaluates the response to treatment within the last 10 days. It is a 5-point scale, with the options " 0 , no good at all" (ineffective treatment), "1, poor" (some effect but unsatisfactory), "2, fair" (reasonable effect but could be better), "3, good" (satisfactory effect with occasional episodes of pain or stiffness), "4, excellent" (ideal response, virtually painfree).$^{32}$ The PGIC is designed to assess patients' perception of change of their global health state (symptoms and quality of life) following treatment within the past 10 days. ${ }^{33,34}$ It is a 7-point scale, with the options " 1 , very much worsened", "2, much worsened", " 3 , minimally worsened", "4, no change", " 5 , minimally improved", " 6 , much improved", and "7, very much improved". The LAFI includes 11 items grouped in three dimensions: Pain (five items), Maximum distance walked (two items), and Activities of daily living (four items). ${ }^{35}$ A score is attributed to each item, and the score per dimension is calculated by adding together the scores for all items of the dimension. The score ranges between 0 and 8 for each dimension, and the LAFI score, which is calculated by adding the score of the three dimensions, between 0 and 24 . Using the LAFI, patients are categorized into six classes: 0 , no handicap $(0) ; 1-4$, mild handicap (1-4); 5-7, moderate handicap; 8-10, severe handicap; $11-13$, very severe handicap; $\geq 14$, extremely severe handicap.

In addition, patients completed a list of pain descriptors elaborated by Cedraschi et al to help define subgroups of OA patients based on pain phenotype. ${ }^{36}$ The 17 items assessed pain quality, investigating Pain sensation (six items), OA-related symptoms (four items), Pain variations (four items), Pain-triggering factors (one item), and Pain and physical activity (two items) (the complete list of items is presented in Figure 2). For each item, the patient attributed a score ranging from 0 to 10 : the higher the score, the greater the intensity. Patients were categorized into clusters according to their response to these items at baseline; the response to the dietary supplementation was analyzed per cluster throughout the study.

In addition, patients completed a 10-cm VAS on Day 0,10 , 20, 30, 60, and 90 for pain assessment, and the DN4 (douleur neuropathique en 4 questions) at inclusion. The DN4 was completed with the GP, and aimed to estimate the likelihood of neuropathic pain; neuropathic pain was probable if the DN4 score was $\geq 4 .{ }^{37}$ Patients were also to provide information on 
the use of concomitant analgesics or anti-inflammatory drugs on Days $0,30,60$, and 90, and on compliance on Days 30, 60, and 90 . Compliance was satisfactory if the patients claimed to have taken at least one capsule per day.

Finally, safety data were collected for the whole study period using a closed question: "was the product welltolerated? Yes, Moderately, Not at all".

\section{Sample Size Calculation and Statistical Analysis}

Based on the McNemar test, with a confidence interval of $95 \%$ and a precision of $10 \%, 96$ patients were required to demonstrate the significant improvement in $50 \%$ of the included patients. Improvement was based on the change in PASS from negative to positive on Day 30. Assuming that about $30 \%$ of the e-CRF would not be assessable, about 126 patients were required to be included in the study.

All statistical analyses were performed using SAS 9.4 (SAS Institute Inc, Cary, NC, US).

Descriptive statistics were provided for the IntentionTo-Treat (ITT) population (ie, all patients who took at least one capsule of dietary supplement) and by a cluster of patients. Patients were categorized into four clusters according to the score they attributed at baseline to each item of the list of pain descriptors elaborated by Cedraschi et $\mathrm{al}^{36}$ using a k-mean clustering analysis.

Continuous variables are presented as mean and standard deviation (SD), and categorical variables as percentages. Repeated measures ANOVA (analysis of variance) was conducted to compare quantitative scores at different time points, while GEE (Generalized estimating equations) method was used for discrete variables and Cochran Q test for binary variables. Intra-group comparisons between two time points were done using paired $t$-test or Wilcoxon signed-rank test (for non-normal distributions) and by McNemar test for binary variables. Comparison between sub-groups was performed using Student's $t$-test or Wilcoxon rank-sum test (for non-normal distributions). Correlations between scores were assessed by Spearman rank-order correlation coefficients.

Univariate and multivariate binary logistic regressions were performed to identify predictive factors of treatment response.

\section{Results}

From 12 March 2017 to 01 March 2019, 123 patients were included in the study: 28 GPs recruited one to 17 patients, each. The last visit of the last patient occurred on 10 July 2019. Of the 123 included patients, 118 took at least one capsule of the dietary supplement; they constituted the ITT population. Among the five patients not included in the ITT population, three were excluded for not meeting the inclusion or non-inclusion criteria, one had not taken any dose of the supplement, and one was a self-included GP. Mean (SD) study follow-up lasted 100.7 (54.9) days per patient.

\section{Baseline Characteristics}

The 118 patients, mainly women (69.5\%), were aged between 42 and 92 years, with a mean age (SD) of 62.9 (9.5) years (Table 1). Their mean BMI was $26.4(4.0) \mathrm{kg} /$ $\mathrm{m} 2 ; 58.5 \%$ of patients had BMI between 25.0 and $35.0 \mathrm{~kg} /$ $\mathrm{m} 2$, indicating that patients were commonly overweight or slightly obese. Pain usually had non-neuropathic characteristics: DN4 score $<4$ in $90.7 \%$ of the patients. Its intensity was 6.1 (1.9) $\mathrm{cm}$ (mean VAS score). Patients were impaired by their disease: $100 \%$ had a negative PASS and $52.6 \%$ had a LAFI score $\geq 8$, indicating severe, very severe, or extremely severe handicap. At baseline, 51.7\% of patients took at least one medical treatment. Main concomitant treatments included agents acting on the reninangiotensin system, diuretics, analgesics, beta-blocking agents, serum-lipid reducing agents, thyroid therapy and anti-inflammatory and anti-rheumatic drugs.

Out of the 118 patients, 97 fully completed the list of pain descriptors at baseline and were categorized into four clusters according to the score they gave to each item on the list (Figure 1). Pain intensity was generally mild in Cluster I ( $\mathrm{N}=41,42.3 \%$; majority of scores under 4), moderate in Clusters II $(\mathrm{N}=22,22.7 \%)$ and IV $(\mathrm{N}=18$, $18.6 \%$; mix of scores under 4 and between 4 and 7), and severe in Cluster III $(\mathrm{N}=16,16.5 \%$; no score under 4 in this cluster). Pain had no specific characteristics in Cluster I. In Cluster II, it was disabling, limiting activity, and frequently associated with joint stiffness and swelling sensation around the joint (higher scores for the corresponding items). In Cluster III, pain was deep and particularly disabling; it affected the joint but also disseminated around the joint and persisted even without physical activity (scores close to or above 7 for the corresponding items). Finally, in Cluster IV, patients had high scores for "weather-related pain" and "stabbing pain" (ie, short, intense, brutally penetrating pain), suggesting possible pain neuropathic component. Baseline comparison for VAS values $(p=0.002)$ and LAFI score $(p=0.006)$ 
Table I Baseline Characteristics of Patients $(\mathrm{N}=|| \mathrm{8})$

\begin{tabular}{|c|c|c|c|}
\hline \multicolumn{2}{|c|}{ Characteristics } & \multirow{2}{*}{$\begin{array}{l}\begin{array}{l}\text { Mean } \\
(S D)\end{array} \\
62.9(9.5)\end{array}$} & \multirow{2}{*}{$\begin{array}{l}\begin{array}{l}\text { Min- } \\
\text { Max }\end{array} \\
42-92\end{array}$} \\
\hline Age (years) & & & \\
\hline Weight (kg) & & $72.9(13.6)$ & $45-112$ \\
\hline Height $(\mathrm{cm})$ & & $165.8(9.0)$ & $148-190$ \\
\hline BMI (kg/m2) & & $26.4(4.0)$ & $18-36$ \\
\hline \multicolumn{2}{|l|}{ LAFI (score)* } & $7.7(3.9)$ & $0-20$ \\
\hline VAS $^{+}$ & & $6.1(1.9)$ & $0-10^{\ddagger}$ \\
\hline DN4 & & $1.4(1.5)$ & $0-7$ \\
\hline \multicolumn{2}{|c|}{ Characteristics } & \multicolumn{2}{|l|}{ No. (\%) } \\
\hline \multirow[t]{2}{*}{ Sex } & Female & \multicolumn{2}{|l|}{$82(69.5 \%)$} \\
\hline & Male & \multicolumn{2}{|l|}{$36(30.5 \%)$} \\
\hline \multirow[t]{5}{*}{ BMI } & $\begin{array}{l}17.0-18.5 \mathrm{~kg} / \mathrm{m}^{2} \text { (slightly } \\
\text { underweight) }\end{array}$ & \multicolumn{2}{|l|}{ I $(0.8 \%)$} \\
\hline & $18.5-25.0 \mathrm{~kg} / \mathrm{m}^{2}$ (normal) & \multicolumn{2}{|l|}{47 (39.8\%) } \\
\hline & $25.0-30.0 \mathrm{~kg} / \mathrm{m}^{2}$ (overweight) & \multicolumn{2}{|l|}{$50(42.4 \%)$} \\
\hline & $\begin{array}{l}30.0-35.0 \mathrm{~kg} / \mathrm{m}^{2} \text { (moderate } \\
\text { obesity) }\end{array}$ & \multicolumn{2}{|l|}{$19(16.1 \%)$} \\
\hline & $\begin{array}{l}35.0-40.0 \mathrm{~kg} / \mathrm{m}^{2} \text { (morbid } \\
\text { obesity) }\end{array}$ & \multicolumn{2}{|l|}{ I $(0.8 \%)$} \\
\hline PASS & Negative & \multicolumn{2}{|l|}{118 (100\%) } \\
\hline \multirow[t]{5}{*}{$\mathrm{LAFI}^{\dagger}$} & Mild handicap $(I-4)$ & \multicolumn{2}{|l|}{$18(18.6 \%)$} \\
\hline & Moderate handicap (5-7) & \multicolumn{2}{|l|}{$28(28.9 \%)$} \\
\hline & Severe handicap (8-10) & \multicolumn{2}{|l|}{$30(30.9 \%)$} \\
\hline & Very severe handicap $(1 \mathrm{I}-13)$ & \multicolumn{2}{|l|}{$12(12.4 \%)$} \\
\hline & $\begin{array}{l}\text { Extremely severe handicap ( } \geq \\
\text { 14) }\end{array}$ & \multicolumn{2}{|l|}{$9(9.3 \%)$} \\
\hline \multirow[t]{2}{*}{ DN4 } & $<4$ & \multicolumn{2}{|l|}{107 (90.7\%) } \\
\hline & $\geq 4$ & \multicolumn{2}{|l|}{ II (9.3\%) } \\
\hline
\end{tabular}

Notes: Baseline: at inclusion or Day 0 visit. $* 21$ missing data; ${ }^{\dagger} 24$ missing data; ${ }^{\ddagger}$ VAS score $\geq 4$ at rest or $\geq 5$ on movement (walking) was required to be included in the study, however, patients could have a score $<4$ at the time of data collection (baseline).

Abbreviations: BMI, body mass index; DN4, Douleur Neuropathique en 4 questions; LAFI, Lequesne algofunctional index; Max, maximum; MD, missing data; Min, minimum; PASS, Patient Acceptable Symptom Scale; SD, standard deviation; VAS, Visual Analogue Scale.

confirmed the difference in pain and disability between the four clusters (Supplementary Table 1). Patients also significantly differed on gender $(\mathrm{p}=0.049)$, height $(\mathrm{p}=0.037)$, and BMI $(\mathrm{p}=0.028)$, but not on age $(\mathrm{p}=0.897)$ or weight $(\mathrm{p}=0.059)$, although for the latter the $\mathrm{p}$-value was close to the significance threshold. Patients from Cluster II were taller, heavier, and more frequently males $(45.5 \%)$ whereas patients from Cluster III were smaller and more frequently females (87.5\%).

\section{Observed Effects Throughout the Dietary Supplementation Period}

The percentage of patients with positive PASS continuously increased from baseline to Day 90 (Cochran Q test: $\mathrm{p}<0.0001)$. On Day 90, 64.5\% of patients were responders (Table 2). Median time to the first PASS change was 34 days (interquartile range: 20-65 days).

MCII and PGIC scores significantly increased throughout the dietary supplementation period (ANOVA: timeeffect, $\mathrm{p}<0.0001)$. Scores on Day 10 were lower than on the other days, and those on Day 90, higher (Table 2). On Day 90, they reached 3.3/4 (1.2) and 4.4/7 (1.8), respectively. The percentages of patients with an MCII $\geq 3$ or a PGIC $\geq 5$ also increased over time, indicating that the percentages of patients with at least good response to treatment or at least noticeable global improvement increased. On Day 90, percentages reached $68.8 \%$ and $58.4 \%$ for MCII and PGIC, respectively.

Handicap assessed by the LAFI score and pain intensity assessed by the VAS significantly decreased throughout the dietary supplementation period (ANOVA: time-effect, $\mathrm{p}<0.0001$ and $\mathrm{p}<0.0001)$. From baseline to Day 90 , mean scores for the LAFI dropped from 7.7/24 (3.9) to 5.2/24 (3.8) and mean scores for the VAS from 6.1/10 (1.9) to 3.5/ 10 (2.5) (Table 2). Scores on all the other assessment days were significantly lower than those at baseline for VAS and those on Day 10 for the LAFI. The percentage of patients with severe to extremely severe handicap (LAFI score $\geq 8$ ) halved; it was $52.6 \%$ at baseline and $26.7 \%$ on Day 90 . The distribution of patients in the various LAFI classes statistically significantly varied over time (GEE method: $\mathrm{p}=0.0006$ ). Simultaneously, the percentage of patients with a meaningful drop (30\%) in VAS score from baseline increased from $24.1 \%$ on Day 10 to $55.2 \%$ on Day 90 .

The scores of all the items describing pain characteristics decreased throughout the dietary supplementation, indicating improvement regardless of pain characteristics (Figure 2).

Finally, the percentage of patients who took concomitant analgesics (GEE method: $\mathrm{p}<0.0001$ ) but not antiinflammatory drugs (GEE method: $\mathrm{p}=0.2086$ ) decreased 


\begin{tabular}{|c|c|c|c|c|}
\hline Items: Mean (SD) & $\begin{array}{l}\text { Cluster I } \\
\qquad \mathbf{N}=41\end{array}$ & $\begin{array}{l}\text { Cluster II } \\
\qquad \begin{array}{l}\mathrm{N}=22 \\
\end{array}\end{array}$ & $\begin{array}{l}\text { Cluster III } \\
\qquad \mathrm{N}=16\end{array}$ & $\begin{array}{l}\text { Cluster IV } \\
\qquad \mathrm{N}=18\end{array}$ \\
\hline Deep * pain & $2.6(2.4)$ & $5.0(2.8)$ & $6.7(1.8)$ & $5.5(2.6)$ \\
\hline Stabbing pain & $1.5(2.6)$ & $2.0(2.1)$ & $5.9(3.0)$ & $7.0(2.2)$ \\
\hline Pain like electric shocks & $1.2(2.4)$ & $1.2(2.2)$ & $5.2(2.5)$ & $4.3(3.4)$ \\
\hline Crushing pain & $1.0(1.7)$ & $3.7(2.6)$ & $5.9(2.7)$ & $2.6(3.3)$ \\
\hline Burning pain & $0.7(1.5)$ & $3.5(3.4)$ & $4.5(3.2)$ & $3.2(3.9)$ \\
\hline Tingling in the joint & $0.4(1.1)$ & $2.0(2.4)$ & $5.6(3.3)$ & $2.7(3.1)$ \\
\hline Widespread pain & $1.2(1.8)$ & $4.7(2.4)$ & $7.1(1.4)$ & $2.3(2.9)$ \\
\hline Joint blockage/Foreign body sensation & $1.0(1.7)$ & $2.8(2.7)$ & $7.4(2.0)$ & $2.3(2.7)$ \\
\hline Joint stiffness & $3.4(2.5)$ & $5.5(2.4)$ & $7.8(1.4)$ & $5.4(3.4)$ \\
\hline Joint swelling ${ }^{\dagger}$ & $3.0(2.6)$ & $6.0(2.3)$ & $7.6(1.9)$ & $2.7(3.0)$ \\
\hline Weather-related pain & $2.3(2.8)$ & $3.3(2.9)$ & $5.4(3.1)$ & $6.7(3.3)$ \\
\hline Pain even without physical activity & $3.2(2.4)$ & $4.9(2.3)$ & $7.1(1.7)$ & $6.2(2.1)$ \\
\hline Limited activity due to pain & $5.2(2.9)$ & $7.5(1.8)$ & $8.3(1.4)$ & $6.1(3.4)$ \\
\hline
\end{tabular}

Figure I Baseline item scores by cluster ( $\mathrm{N}=97)$. *Without identified precise impact point; ${ }^{\dagger}$ Swelling sensation around the joint. Clusters are based on scores for each item except four items which were excluded due to the high variability of the answers. In red, scores $\geq 7$; in orange, scores between 4.0 and 7.0 ; in green, scores $<4.0$. Abbreviation: $\mathrm{N}$, number of available data.

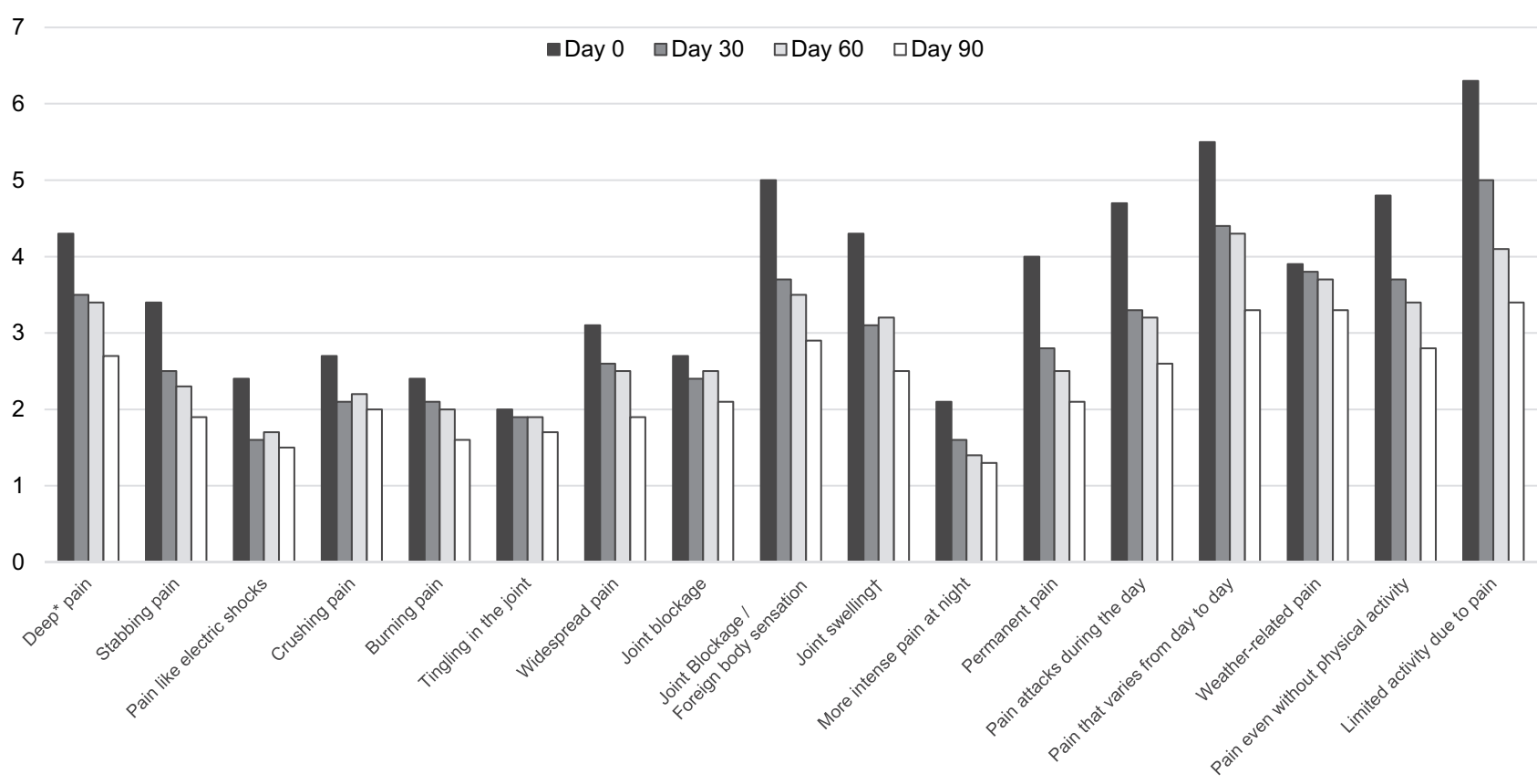

Figure 2 Evolution of mean scores throughout the supplementation, items describing KOA pain. Supplementation: combination of turmeric, boswellia, and red algae extracts. *Without identified precise impact point; ${ }^{\dagger}$ Swelling sensation around the joint.

Abbreviation: KOA, knee osteoarthritis. 
Table 2 Summary of the Effects of Turmeric, Boswellia, and Red Algae Extracts in KOA Patients (N=I18)

\begin{tabular}{|c|c|c|c|c|c|c|c|c|}
\hline Self-Reported Tools & & & Day 0 & Day 10 & Day 20 & Day 30 & Day 60 & Day 90 \\
\hline \multirow[t]{2}{*}{ PASS } & & $\mathrm{N}$ & 118 & 95 & 93 & 94 & 85 & 76 \\
\hline & Positive * & $\%$ & 0 & 43.2 & 49.5 & 42.6 & 63.5 & 64.5 \\
\hline \multirow[t]{3}{*}{$\mathrm{MCll}$} & & $\mathrm{N}$ & - & 95 & 93 & 94 & 85 & 77 \\
\hline & Score ${ }^{\dagger}$ & Mean (SD) & - & $2.5(1.0)$ & $2.8(1.1)$ & $2.9(1.1)$ & $3.1(1.2)$ & $3.3(1.2)$ \\
\hline & Score $\geq 3$ & $\%$ & - & 44.2 & 60.2 & 60.6 & 64.7 & 68.8 \\
\hline \multirow[t]{3}{*}{ PGIC } & & $\mathrm{N}$ & - & 95 & 93 & 94 & 85 & 77 \\
\hline & Score ${ }^{\dagger}$ & Mean (SD) & - & $3.3(1.5)$ & $3.7(1.6)$ & $3.7(1.6)$ & $3.9(1.9)$ & $4.4(1.8)$ \\
\hline & Score $\geq 5$ & $\%$ & - & 27.4 & 38.7 & 35.1 & 42.4 & 58.4 \\
\hline \multirow[t]{2}{*}{ LAFI } & & $\mathrm{N}$ & 97 & - & - & 88 & 85 & 75 \\
\hline & Score ${ }^{\dagger}$ & Mean (SD) & $7.7(3.9)$ & - & - & $6.1(3.8)$ & $6.0(4.3)$ & $5.2(3.8)$ \\
\hline \multirow[t]{5}{*}{ Handicap ${ }^{\ddagger}$} & Mild & $\%$ & 18.6 & - & - & 35.2 & 36.5 & 49.3 \\
\hline & Moderate & $\%$ & 28.9 & - & - & 30.7 & 27.1 & 24.0 \\
\hline & Severe & $\%$ & 30.9 & - & - & 18.2 & 24.7 & 16.0 \\
\hline & Very severe & $\%$ & 12.4 & - & - & 12.5 & 7.1 & 6.7 \\
\hline & Extremely severe & $\%$ & 9.3 & - & - & 3.4 & 4.7 & 4.0 \\
\hline \multirow[t]{2}{*}{ VAS } & & $\mathrm{N}$ & 94 & 90 & 88 & 87 & 79 & 69 \\
\hline & Score $^{\dagger}$ & Mean (SD) & $6.1(1.9)$ & $5.1(1.9)$ & $4.7(2.0)$ & $4.5(2.1)$ & $4.0(2.3)$ & $3.5(2.5)$ \\
\hline \multirow[t]{2}{*}{ Decrease in VAS score from Day 0} & & $\mathrm{~N}$ & - & 87 & 85 & 84 & 76 & 67 \\
\hline & $\geq 30 \%$ & $\%$ & - & 24.1 & 30.6 & 39.3 & 48.7 & 55.2 \\
\hline \multirow[t]{3}{*}{ Concomitant treatments $\S$} & & $\mathrm{N}$ & 98 & - & - & 93 & 85 & 77 \\
\hline & Anti-inflammatory drugs & $\%$ & 30.6 & - & - & 24.7 & 21.2 & 23.4 \\
\hline & Analgesics & $\%$ & 59.2 & - & - & 50.0 & 48.2 & 35.1 \\
\hline
\end{tabular}

Notes: *Cochran $Q$ test, $p<0.000$ I; ANOVA: time-effect ( $p<0.000$ I); a statistically significant difference was found between Baseline or Day 10 score and other assessment days: $p$-values for PGIC on Days 20, 30, 60, and 90 versus Day 10: $p=0.0160, p=0.0091, p=0.0004$, and $p<0.0001$; $p$-values for MCII on Days 20, 30, 60, and 90 versus Day 10: $p=0.0007, p=0.0019, p<0.0001$, and $p<0.0001$; $p$-value for VAS on Days 10, 20, 30, 60, and 90 versus Baseline: $p<0.000$ I; $p$-value for Global score (Lequesne index) on Days 30,60 , and 90 versus Baseline: $p<0.0001$; ${ }^{\ddagger}$ GEE method, $p=0.0006$; ${ }^{\S}$ GEE method, $p=0.2086$ for anti-inflammatory drugs and $p<0.0001$ for analgesics.

Abbreviations: KOA, knee osteoarthritis; LAFI, Lequesne algofunctional index; MCII, Minimal Clinically Important Improvement; N, number of patients; PASS, Patient Acceptable Symptom Scale; PGIC, Patient Global Impression of Change; SD, standard deviation; VAS, Visual Analogue Scale.

between Day 0 and Day 90. On Day $90,35.1 \%$ of patients (versus $59.2 \%$ on Day 0 ) reported taking analgesics in the previous month (Table 2).

\section{Factors Associated with Treatment Response}

Univariate analyses were carried out to identify potential baseline factors associated with positive PASS (ie, treatment response) on Day 30, which was close to the median time to first PASS change (34 days). Overall, 15 variables were identified $(\mathrm{p}<0.1)$. Patients with low LAFI or VAS scores at baseline were more likely to be responders: the odds ratio (OR) was 0.853 (95\% confidence interval [CI]: 0.751-0.968; $\mathrm{p}=0.0137)$ for the LAFI and 0.739 (95\% CI: $0.58-0.942$; $\mathrm{p}=0.0146$ ) for the VAS (Supplementary Table 2). Patients with a low score for 12 of the 17 items at baseline were also more likely to be responders on Day 30. In addition, responders tended to less frequently take concomitant analgesic drugs $(\mathrm{p}=0.0662)$ but not anti-inflammatory drugs $(\mathrm{p}=0.3581)$. Age, gender, weight, height, BMI, DN4 score 
were not identified as independent factors associated with treatment response (Supplementary Table 2).

Multivariate analysis showed that "Stabbing pain" and "Widespread pain" were significant factors associated with treatment response. Patients with low scores were more likely to be responders. The odds ratios (ORs) were 0.820 (95\% CI: 0.705-0.954) and 0.737 (0.614-0.884) for "Stabbing pain" and "Widespread pain", respectively (first model including the 15 variables identified in the univariate analysis). A second model excluding pain descriptors highlighted VAS as a significant factor associated with treatment response. The OR was $0.739(95 \%$ CI: 0.580-0.942) for the VAS meaning that with a VAS score 1 point lower at inclusion, patients were 1.4 times more likely to respond to treatment at Day 30 .

\section{Observed Effects by Cluster of Patients}

Data from PROMs were analyzed according to the cluster of patients. Responders were observed in all clusters with variations in response. From Day 10 to Day 90, the percentage of patients with positive PASS (PASS+) statistically significantly and continuously increased in Clusters I and IV (Cochran Q test, $\mathrm{p}=0.0052$ and $\mathrm{p}=0.0187$, respectively). MCII and PGIC scores statistically significantly increased over time in Clusters I and IV (ANOVA, time effect: $\mathrm{p}=0.0157$ and $\mathrm{p}<0.0001$ ). Better improvement or relief was reported in Cluster I and Cluster IV for PASS, MCII (percentage of patients with $\mathrm{MCII} \geq 3$ ), PGIC (percentage of patients with PGIC $\geq 5$ ), and VAS (percentage of patients with VAS score drop $\geq 30 \%$ ). The observed improvement tended to be continuous over the 90 days of treatment in patients from Cluster I regardless of the PROMs (Figure 3).

A statistically significant relief was found in disability (LAFI) and pain (VAS score) in the four clusters (ANOVA, time effect: $\mathrm{p}<0.05$ ). The percentage of patients mildly disabled statistically significantly increased from baseline to Day 90 in Clusters II and IV (ANOVA: time effect, $p=0.0007$ and $p=0.0458$ ), but not in the other clusters $(\mathrm{p}>0.05)$. The percentage of patients with mild handicap (LAFI score $<8$ ) increased in all clusters whereas the percentage of patients severely, very severely, or extremely severely disabled (LAFI score $>11$ ) decreased (Figure 4).

Around the date of the first change in PASS (Day 30), the percentage of responders was $62.2 \%, 36.4 \%, 14.3 \%$ and $22.2 \%$, for Clusters I, II, III, and IV, respectively. According to univariate analyses (Supplementary Table 2), patients from Cluster I but not those from Clusters II or IV were more likely to respond than those from Cluster III $(p=0.0058)$.

\section{Safety and Compliance}

Safety data were reported for 77 of the 118 patients. Out of these patients, 74 (96.1\%) claimed that the dietary supplement was well tolerated. The three patients who reported inconveniences (eg, meteorism) did not prematurely withdraw from the study.

Treatment compliance was satisfactory: 87 (92.6\%), 78 $(91.8 \%)$, and $67(88.2 \%)$ of the patients claimed to have taken at least one capsule each day for 30, 60, and 90 days.

\section{Discussion}

The present study found a noticeable relief, as assessed by PROMs, in KOA patients who took oral dietary supplementation with a fixed combination of boswellia, turmeric, and red algae extracts. All assessed measures systematically showed improvement. The present study also showed that half of the patients reported relief after approximately one month of dietary supplementation. Finally, different profiles of KOA patients were identified, some being more improved by the dietary supplement than the others, and also predictive factors of the response.

The ability of boswellia (Boswellia serrata) and turmeric (Curcuma longa) extracts (alone or in combination) to relieve patients with KOA has been largely described in the literature. ${ }^{9-20}$ However, to the best of our knowledge, this is the first and unique study reporting data where their combination with red algae extracts is used. Evaluating the effect of the fixed combination of boswellia, turmeric, and red algae extracts was necessary as the active ingredients contained in the different extracts may act synergistically. ${ }^{38}$

The dietary supplement relieved patients and decreased pain intensity by $30 \%$ or more in $55 \%$ of patients. In clinical practice, as the fixed combination of boswellia, turmeric, and red algae extracts is well tolerated and no contraindications with antalgics exist, the dietary supplement can be used alone or with anti-inflammatory or antalgic drugs if required. Pain relief was observed after 34 days of supplementation (median time for positive PASS), suggesting that at least one-month treatment was required to evaluate the impact of the dietary supplement in KOA patients. Relief was maximal after 90 days; it was continuous and significant mainly in patients from Cluster I: ie, patients with pain of mild intensity and without specific characteristics. However, responders were 


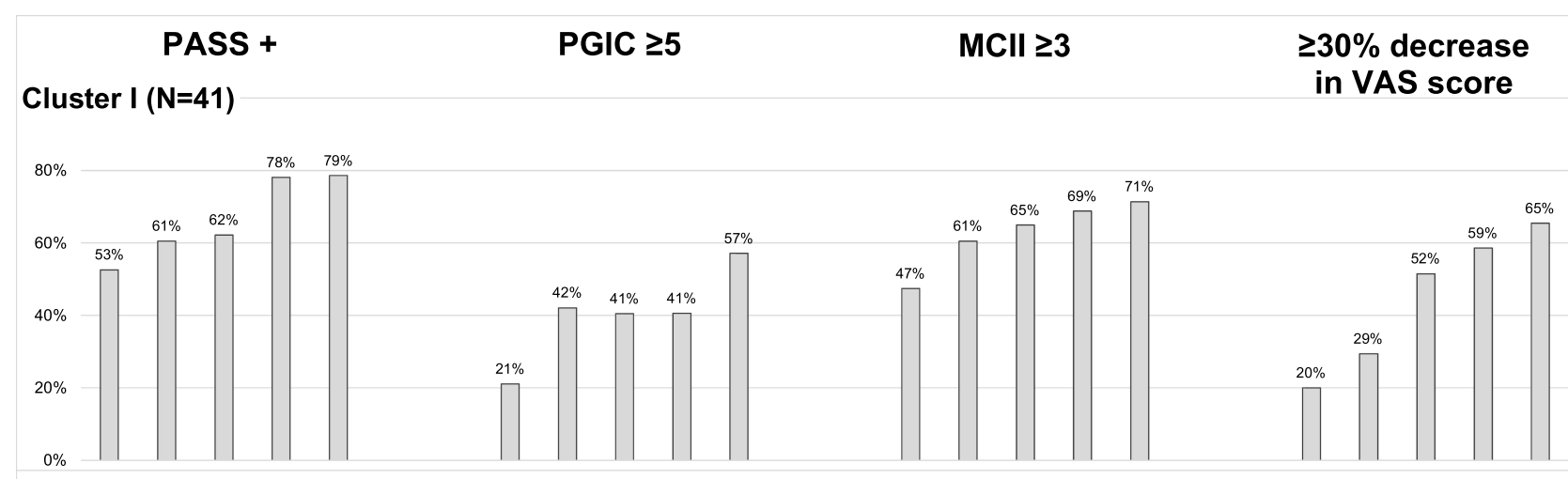

\section{Cluster II ( $\mathrm{N}=\mathbf{2 2})$}

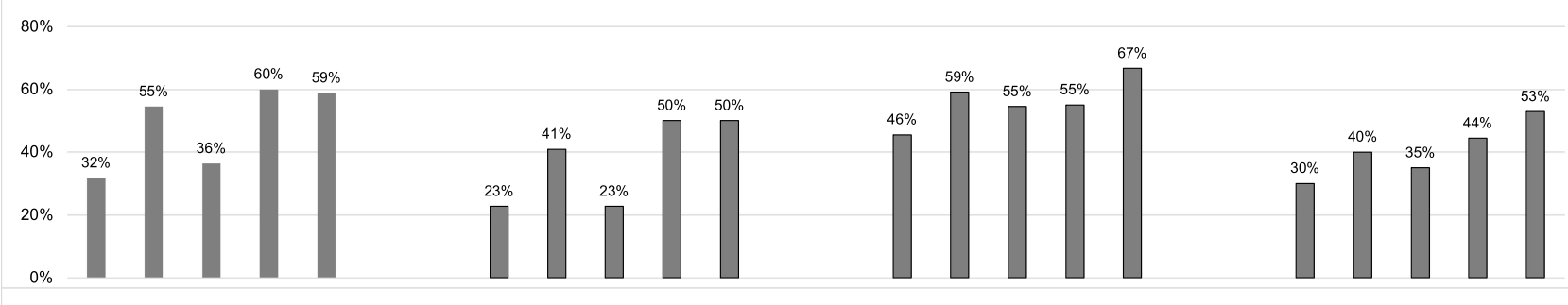

\section{Cluster III ( $\mathrm{N}=16)$}

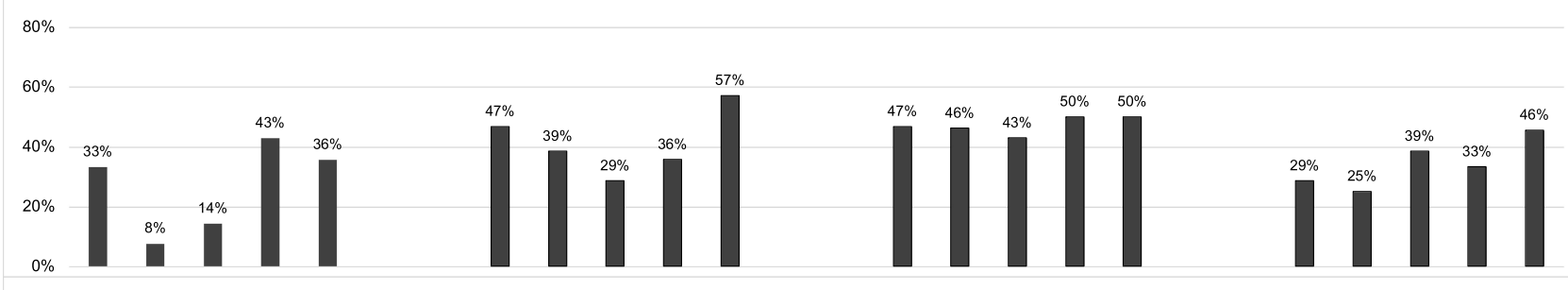

\section{Cluster IV (N=18)}

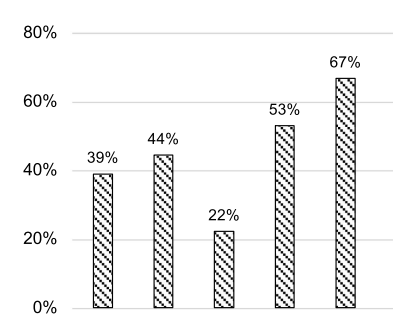

Day $10 \quad 20 \quad 30 \quad 60 \quad 90$

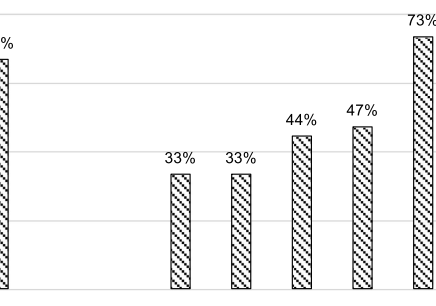

Day $10 \quad 20 \quad 30 \quad 60 \quad 90$

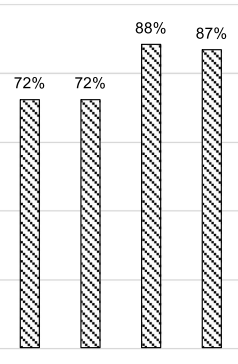

Day $10 \quad 20 \quad 30 \quad 60 \quad 90$

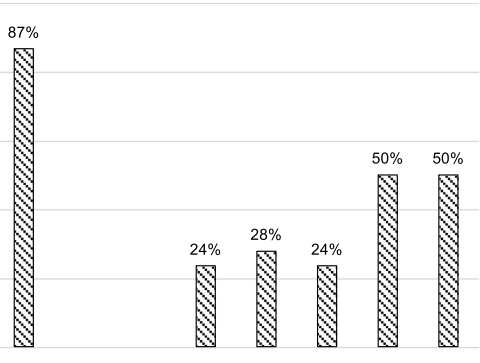

Day $10 \quad 20 \quad 30 \quad 60 \quad 90$

Figure 3 Changes in PASS, PGIC, MCII, and VAS throughout the supplementation period by cluster. Percentages of patients are presented for each parameter and by Days in each cluster. Supplementation: combination of turmeric, boswellia, and red algae extracts. For the characteristics of the patients please see Supplementary Table I.

observed in all clusters, showing a benefit from using the dietary supplement in patients with more severe symptoms. Altogether, these results suggest that the dietary supplement should be used at an early stage and for at least three months.

Most studies evaluating treatment effect in KOA usually focused on pain intensity and physical function. ${ }^{9-20}$ In these studies, pain intensity was commonly assessed using a VAS and physical function using the Western Ontario McMaster Arthritis Index (WOMAC), a self-administered 24-item questionnaire. ${ }^{10,12,13,15-17,20}$ The WOMAC assessed activities of daily living, functional mobility, gait, general health and quality of life. PROMs are useful to provide data on all aspects of the disease and its treatment as perceived by the 


\section{LAFI: Mild Moderate Severe*}

\section{Cluster I $(\mathrm{N}=\mathbf{4 1})$}

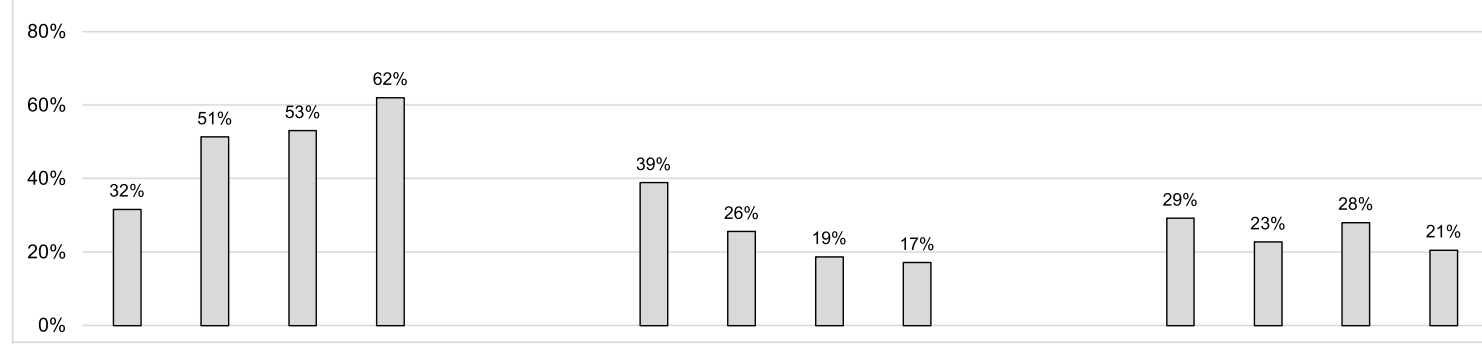

\section{Cluster II ( $\mathbf{N = 2 2 )}$}

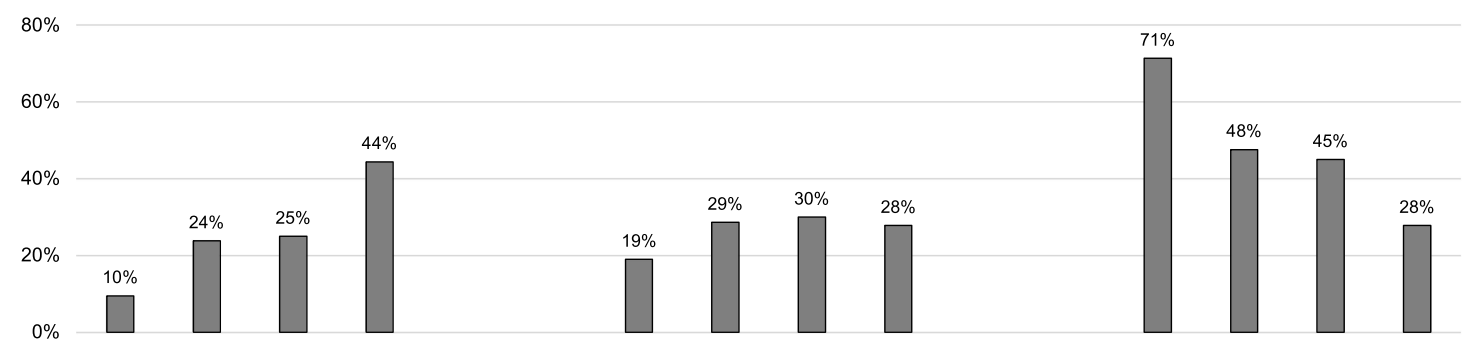

\section{Cluster III $(\mathrm{N}=16)$}

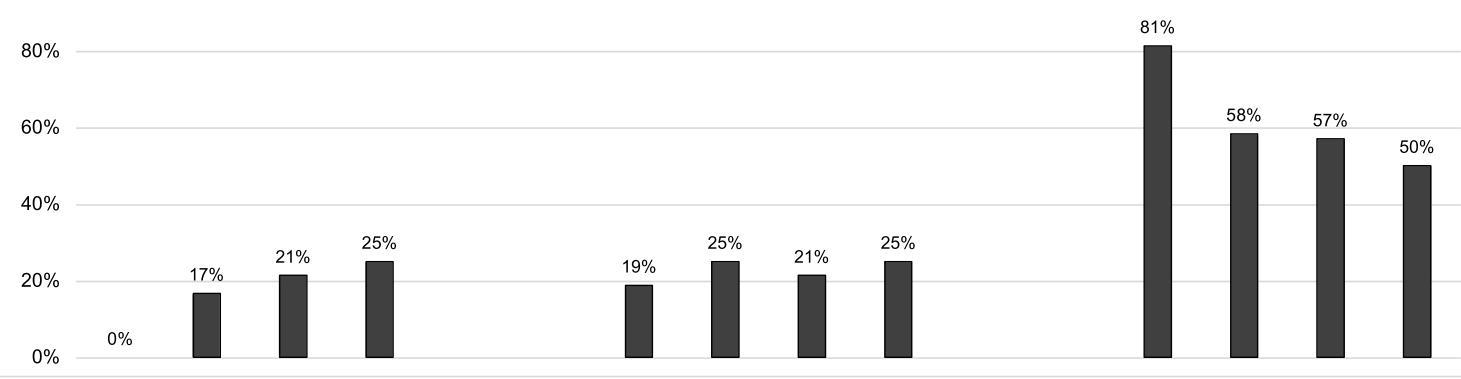

\section{Cluster IV ( $\mathrm{N}=18)$}

$80 \%$

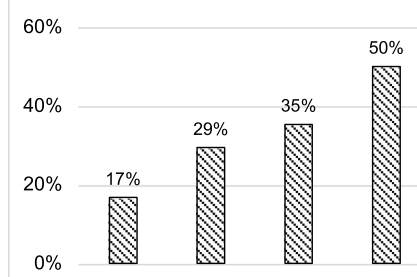

Day $10 \quad 30 \quad 60 \quad 90$
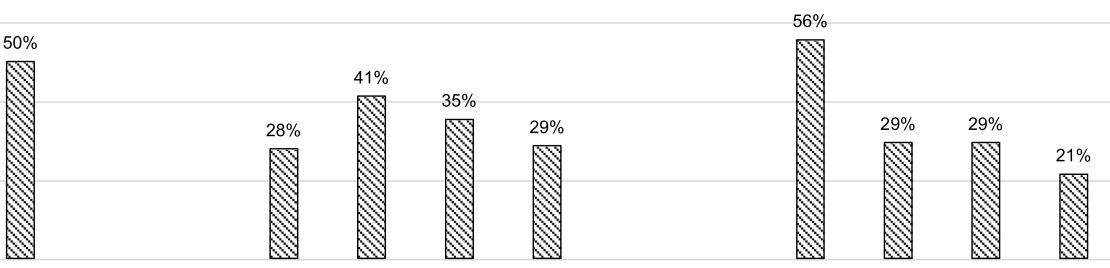

Day $10 \quad 30 \quad 60 \quad 90$

Day $10 \quad 30 \quad 60 \quad 90$

Figure 4 Over time changes in handicap severity (Lequesne index) by cluster. *Percentages for severe, very severe and extremely severe handicap are grouped. Percentages of patients are presented for each parameter and by Days in each cluster. For the characteristics of the patients please see Supplementary Table I.

Abbreviation: LAFI, Lequesne algofunctional index. 
patients. Moreover, they limit observer bias. ${ }^{38}$ They are particularly useful to assess change in chronic conditions. However, the WOMAC does not focus on pain whereas $\mathrm{KOA}$ is a painful chronic condition and pain is a complex and subjective experience; its quantification is challenging. As currently no reliable method objectively quantifies patient's experience of pain, we used a battery of PROMs, which is the best way to estimate pain intensity, pain quality, and/or pain relief following treatment as perceived by the patients. $^{39}$

In addition to PROMs, we used a list of pain descriptors for assessment of pain quality because KOA cannot be reduced to pain intensity, and the fixed combination of boswellia, turmeric, and red algae extracts is not an antalgic drug but a combination of active ingredients with anti-inflammatory and/or anti-nociceptive properties. The PROMs and the list of pain descriptors were useful to provide data on all aspects of the disease and its management by a non-antalgic drug, as perceived by the patients. In addition, pain descriptors can be useful to identify the profiles of KOA patients who could be relieved by the dietary supplement.

This study presented the limitations of any open-label observational study, the main one being the absence of a control group. Therefore, the effects of the combination tested might have been overestimated. The promising results observed in this preliminary study will have to be confirmed in a controlled study. It also included the potential Hawthorne effect, although this effect would have probably decreased over time. Finally, a potential bias due to the number of missing data at each visit cannot be ruled out, in particular on Day 90. Indeed, as according to the visit and the PROMs, up to $30 \%$ of the data were missing (as expected for an observational study), the results may have been overestimated. However, even assuming that all missing data were treatment failures (non-responding patients), which was not the case (data not shown), a noticeable proportion of patients would have been relieved.

\section{Conclusion}

Although its results need to be confirmed in further studies and, in particular, a randomized placebo-controlled study, this preliminary study suggests that the fixed combination of Boswellia serrata, Curcuma longa, and Porphyra umbilicalis extracts could relieve KOA patients. This study also identified four clusters of patients according to pain characteristics. Responders were observed in the four clusters with better relief reported by the cluster of patients with pain of mild intensity and without specific characteristics, suggesting the interest of this dietary supplementation in patients with early disease. Although the benefits of the dietary supplement were maximal after 90 days of treatment, these benefits could be observed earlier; nevertheless, in clinical practice, dietary supplementation should be maintained for one to three months before ruling out its ability to relieve KOA. None of the sociodemographic or anthropomorphic characteristics of the patients predicted treatment response, whereas VAS score and two pain characteristics were predictive factors for treatment response. Further studies are needed to better identify profiles of KOA patients for whom the dietary supplement would be useful. Beyond these results, this study, therefore, showed the importance of PROs and specific pain qualitative descriptors for the accurate evaluation of dietary supplement approaches in painful conditions.

\section{Abbreviations}

ANOVA, analysis of variance; BMI, body mass index; CI, confidence interval; DN4, Douleur Neuropathique en 4 questions; e-CRF, electronic case report form; GEE, Generalized estimating equations; GP, general practitioner; ITT, Intention-To-Treat; KOA, knee osteoarthritis; LAFI, Lequesne algofunctional index; MCII, Minimal Clinically Important Improvement; MD, missing data; $\mathrm{N}$, number of patients; NSAID, non-steroidal anti-inflammatory drug; OA, osteoarthritis; OTC, over the counter; PASS, Patient Acceptable Symptom Scale; PGIC, Patient Global Impression of Change; PROM, patient-reported outcome measure; SD, standard deviation; STROBE, Strengthening the Reporting of Observational studies in Epidemiology VAS, Visual Analogue Scale; WOMAC, Western Ontario McMaster Arthritis Index.

\section{Data Sharing Statement}

The data that support the findings of this study are available from PiLeJe (37 quai de Grenelle, 75015 Paris, France) but restrictions apply to the availability of these data, which were used under license for the current study, and so are not publicly available. Data are however available from the authors (Samira Ait Abdellah: s.aitabdellah@pileje.com) upon reasonable request and with permission of PiLeJe.

\section{Ethics Approval and Consent to Participate}

The present study was conducted according to the French law and guidelines on non-interventional studies in force when the study was launched. The protocol was approved 
by the French Committee for Health Research Data Processing, CCTIRS (Comité consultatif sur le traitement de l'information en matière de recherche dans le domaine de la santé) on 16 September 2016 (No: 16-634). Following a commitment to comply with relevant legislation and regulations, a declaration of conformity was made to the French national commission on personal data privacy, CNIL (Commission Nationale de l'Informatique et des Libertés). In compliance with the French regulation for observational studies, all patients included in the study freely provided verbal informed consent to participate. Verbal informed consent was approved by the French Committee for Health Research Data Processing.

\section{Acknowledgments}

The authors would like to thank K. André (Statitec) for statistical analyses. They also thank M. Chanard and F. Péretz (Abelia Science), and C. Blondeau (PiLeJe Laboratoire) for their help in preparing the manuscript.

\section{Author Contributions}

All authors made a significant contribution to the work reported, whether that is in the conception, study design, execution, acquisition of data, analysis and interpretation, or in all these areas; took part in drafting, revising or critically reviewing the article; gave final approval of the version to be published; have agreed on the journal to which the article has been submitted; and agree to be accountable for all aspects of the work.

\section{Disclosure}

S. Perrot reports grants and personal fees from PiLeJe. A. P. Trouvin reports that she has no competing interests to declare. S. Ait-Abdellah, C. Gal, and A. Leblanc are employees of PiLeJe Laboratoire. The authors report no other conflicts of interest in this work.

\section{References}

1. Osteoarthritis Research Society International (OARSI). Osteoarthritis: a serious disease, submitted to the US food and drug administration. 2016. Available from: https://www.oarsi.org/sites/default/files/library/2018/pdf/ oarsi_white_paper_oa_serious_disease121416_1.pdf. Accessed October 13, 2020.

2. Vina ER, Kwoh CK. Epidemiology of osteoarthritis: literature update. Curr Opin Rheumatol. 2018;30(2):160-167. doi:10.1097/BOR.0000000000 000479.

3. Bannuru RR, Osani MC, Al-Eid F, Wang C. Efficacy of curcumin and Boswellia for knee osteoarthritis: systematic review and meta-analysis. Semin Arthritis Rheum. 2018;48(3):416-429. doi:10.1016/j.semarthrit. 2018.03.001.
4. Glyn-Jones S, Palmer AJ, Agricola R, et al. Osteoarthritis. Lancet. 2015;386(9991):376-387. doi:10.1016/S0140-6736(14)60802-3.

5. Bannuru RR, Osani MC, Vaysbrot EE, et al. OARSI guidelines for the non-surgical management of knee, hip, and polyarticular osteoarthritis. Osteoarthritis Cartilage. 2019;27(11):1578-1589. doi:10.1016/j. joca.2019.06.011.

6. Conaghan PG, Arden N, Avouac B, Migliore A, Rizzoli R. Safety of paracetamol in osteoarthritis: what does the literature say? Drugs Aging. 2019;36(Suppl 1):7-14. doi:10.1007/s40266-01900658-9.

7. Kingsbury SR, Gross HJ, Isherwood G, Conaghan PG. Osteoarthritis in Europe: impact on health status, work productivity and use of pharmacotherapies in five European countries. Rheumatology (Oxford). 2014;53(5):937-947. doi:10.1093/rheumatology/ket463.

8. Delouya S, Crosnier G, Lacombe J, Desericourt C, Milon JY. Pharmacist-led medication for pain in France: what population are we talking about? Results of the Optymed study. Presse Med. 2019;48(10):e273-e283. doi:10.1016/j.lpm.2019.07.038.

9. Kimmatkar N, Thawani V, Hingorani L, Khiyani R. Efficacy and tolerability of Boswellia serrata extract in treatment of osteoarthritis of knee-a randomized double blind placebo controlled trial. Phytomedicine. 2003;10(1):3-7. doi:10.1078/094471103321648593

10. Sengupta K, Alluri KV, Satish AR, et al. A double blind, randomized, placebo controlled study of the efficacy and safety of 5-Loxin for treatment of osteoarthritis of the knee. Arthritis Res Ther. 2008;10(4): R85. doi:10.1186/ar2461.

11. Gupta PK, Samarakoon SM, Chandola HM, Ravishankar B. Clinical evaluation of Boswellia serrata (Shallaki) resin in the management of Sandhivata (osteoarthritis). Ayu. 2011;32(4):478-482. doi:10.4103/ 0974-8520.96119.

12. Majeed M, Majeed S, Narayanan NK, Nagabhushanam K. A pilot, randomized, double-blind, placebo-controlled trial to assess the safety and efficacy of a novel Boswellia serrata extract in the management of osteoarthritis of the knee. Phytother Res. 2019;33 (5):1457-1468. doi:10.1002/ptr.6338.

13. Belcaro G, Cesarone MR, Dugall M, et al. Product-evaluation registry of Meriva ${ }^{\circledR}$, a curcumin-phosphatidylcholine complex, for the complementary management of osteoarthritis. Panminerva Med. 2010;52(2 Suppl 1):55-62.

14. Appelboom T, Maes N, Albert A. A new curcuma extract $\left(\right.$ flexofytol $^{\circledR}$ ) in osteoarthritis: results from a belgian real-life experience. Open Rheumatol J. 2014;8:77-81. doi:10.2174/ 1874312901408010077.

15. Kuptniratsaikul V, Dajpratham P, Taechaarpornkul W, et al. Efficacy and safety of Curcuma domestica extracts compared with ibuprofen in patients with knee osteoarthritis: a multicenter study. Clin Interv Aging. 2014;9:451-458. doi:10.2147/CIA.S58535.

16. Srivastava S, Saksena AK, Khattri S, Kumar S, Dagur RS. Curcuma longa extract reduces inflammatory and oxidative stress biomarkers in osteoarthritis of knee: a four-month, double-blind, randomized, placebo-controlled trial. Inflammopharmacology. 2016;24(6):377388. doi:10.1007/s10787-016-0289-9.

17. Panda SK, Nirvanashetty S, Parachur VA, Mohanty N, Swain T. A randomized, double blind, placebo controlled, parallel-group study to evaluate the safety and efficacy of Curene ${ }^{\mathbb{R}}$ versus placebo in reducing symptoms of knee OA. Biomed Res Int. 2018;2018:5291945. doi: $10.1155 / 2018 / 5291945$.

18. Henrotin Y, Malaise M, Wittoek R, et al. Bio-optimized Curcuma longa extract is efficient on knee osteoarthritis pain: a double-blind multicenter randomized placebo controlled three-arm study. Arthritis Res Ther. 2019;21(1):179. doi:10.1186/s13075-019-1960-5.

19. Kizhakkedath R. Clinical evaluation of a formulation containing Curcuma longa and Boswellia serrata extracts in the management of knee osteoarthritis. Mol Med Rep. 2013;8(5):1542-1548. doi:10. 3892/mmr.2013.1661. 
20. Haroyan A, Mukuchyan V, Mkrtchyan N, et al. Efficacy and safety of curcumin and its combination with boswellic acid in osteoarthritis: a comparative, randomized, double-blind, placebo-controlled study. BMC Complement Altern Med. 2018;18(1):7. doi:10.1186/s12906-017-2062-z.

21. Abdel-Tawab M, Werz O, Schubert-Zsilavecz M. Boswellia serrata: an overall assessment of in vitro, preclinical, pharmacokinetic and clinical data. Clin Pharmacokinet. 2011;50(6):349-369. doi:10.2165/ 11586800-000000000-00000.

22. Sengupta K, Kolla JN, Krishnaraju AV, et al. Cellular and molecular mechanisms of anti-inflammatory effect of Aflapin: a novel Boswellia serrata extract. Mol Cell Biochem. 2011;354(1-2):189-197. doi:10.1007/s11010-011-0818-1.

23. Umar S, Umar K, Sarwar AH, et al. Boswellia serrata extract attenuates inflammatory mediators and oxidative stress in collagen induced arthritis. Phytomedicine. 2014;21(6):847-856. doi:10.1016/j.phymed.2014.02. 001.

24. Deodhar SD, Sethi R, Srimal RC. Preliminary study on antirheumatic activity of curcumin (diferuloyl methane). Indian J Med Res. 1980;71:632-634.

25. Shen CL, Smith BJ, Lo DF, et al. Dietary polyphenols and mechanisms of osteoarthritis. J Nutr Biochem. 2012;23(11):1367-1377. doi:10.1016/j.jnutbio.2012.04.001.

26. Silva LM, Lima V, Holanda ML, et al. Antinociceptive and antiinflammatory activities of lectin from marine red alga Pterocladiella capillacea. Biol Pharm Bull. 2010;33(5):830-835. doi:10.1248/ bpb.33.830.

27. Quinderé AL, Fontes BP, Vanderlei Ede S, et al. Peripheral antinociception and anti-edematogenic effect of a sulfated polysaccharide from Acanthophora muscoides. Pharmacol Rep. 2013;65(3):600613. doi:10.1016/s1734-1140(13)71037-5

28. Batista JA, Dias EG, Brito TV, et al. Polysaccharide isolated from Agardhiella ramosissima: chemical structure and anti-inflammation activity. Carbohydr Polym. 2014;99:59-67. doi:10.1016/j.carbpol.2013. 08.071 .

29. Pereira JG, Mesquita JX, Aragão KS, et al. Polysaccharides isolated from Digenea simplex inhibit inflammatory and nociceptive responses. Carbohydr Polym. 2014;108:17-25. doi:10.1016/j. carbpol.2014.01.105.

30. Birnie KA, McGrath PJ, Chambers CT. When does pain matter? Acknowledging the subjectivity of clinical significance. Pain. 2012;153(12):2311-2314. doi:10.1016/j.pain.2012.07.033.

31. Dougados M, Luo MP, Maksymowych WP, et al; ATLAS STUDY GROUP. Evaluation of the patient acceptable symptom state as an outcome measure in patients with ankylosing spondylitis: data from a randomized controlled trial. Arthritis Rheum. 2008;59(4):553-560. doi:10.1002/art.23527.
32. Tubach F, Ravaud P, Baron G, et al. Evaluation of clinically relevant changes in patient reported outcomes in knee and hip osteoarthritis: the minimal clinically important improvement. Ann Rheum Dis. 2005;64(1):29-33. doi:10.1136/ard.2004.022905.

33. Guy W. ECDEU Assessment Manual for Psychopharmacology. Rockville, Md: U.S. Dept. of Health, Education, and Welfare, Public Health Service, Alcohol, Drug Abuse, and Mental Health Administration, National Institute of Mental Health, Psychopharmacology Research Branch, Division of Extramural Research Programs; 1976.

34. Farrar JT, Young JP, LaMoreaux L, Werth JL, Poole RM. Clinical importance of changes in chronic pain intensity measured on an 11point numerical pain rating scale. Pain. 2001;94:149-158. doi:10.1016/S0304-3959(01)00349-9

35. Lequesne MG. The algofunctional indices for hip and knee osteoarthritis. J Rheumatol. 1997;24(4):779-781.

36. Cedraschi C, Delézay S, Marty M, et al. "Let's talk about OA pain": a qualitative analysis of the perceptions of people suffering from OA. Towards the development of a specific pain OArelated questionnaire, the osteoarthritis symptom inventory scale (OASIS). PLoS One. 2013;8(11):e79988. doi:10.1371/journal. pone.0079988.

37. Bouhassira D, Attal N, Alchaar H, et al. Comparison of pain syndromes associated with nervous or somatic lesions and development of a new neuropathic pain diagnostic questionnaire (DN4). Pain. 2005;114:29-36. doi:10.1016/j.pain.2004.12.010

38. Mobasheri A, Henrotin Y. Comment on: efficacy of Curcumin and Boswellia for knee osteoarthritis: systematic review and meta-analysis. Semin Arthritis Rheum. 2019;48(4):e25-e26. doi:10.1016/j. semarthrit.2018.04.002

39. Sørensen NL, Hammeken LH, Thomsen JL, Ehlers LH. Implementing patient-reported outcomes in clinical decision-making within knee and hip osteoarthritis: an explorative review. $B M C$ Musculoskelet Disord. 2019;20(1):230. doi:10.1186/s12891-0192620-2.

40. Perrot S, Lantéri-Minet M. Patients' Global Impression of Change in the management of peripheral neuropathic pain: clinical relevance and correlations in daily practice. Eur J Pain. 2019;23(6):1117-1128. doi:10.1002/ejp.1378.
Open Access Rheumatology Research and Reviews is an international, peer-reviewed, open access journal publishing original research, reports, editorials, reviews and commentaries on all aspects of clinical and experimental rheumatology in the clinic and laboratory including the following topics: Pathology, pathophysiology of rheumatological diseases; Investigation, treatment and management of rheumatological diseases; Clinical trials and novel pharmacological approaches for the treatment of rheumatological disorders. The manuscript management system is completely online and includes a very quick and fair peer-review system, which is all easy to use. Visit http://www.dovepress.com/testimonials.php to read real quotes from published authors 